\title{
CONSTRUÇÃO CIENTÍFICA DA ATUAÇÃO DAS \\ ENFERMEIRAS EM PANDEMIAS: REVISÃO INTEGRATIVA
}

\author{
SCIENTIFIC CONSTRUCTION OF NURSES' WORK \\ DURING PANDEMICS: INTEGRATIVE REVIEW
}

\section{CONSTRUCCIÓN CIENTÍFICA DEL TRABAJO DE LAS ENFERMERAS EN PANDEMIAS: REVISIÓN INTEGRATIVA}

\author{
Deybson Borba de Almeida ${ }^{1}$ \\ Laiane da Silva Santana ${ }^{2}$ \\ Maria Talita Cruz Silva Oliveira ${ }^{3}$ \\ Nívia Vanessa Carneiro dos Santos \\ Igor Ferreira Borba de Almeida \\ Caio Moura dos Santos
}

Como citar este artigo: Almeida DB, Santana LS, Oliveira MTCS, Santos NVC, Almeida IFB, Santos CM. Construção científica da atuação das enfermeiras em pandemias: revisão integrativa. Rev baiana enferm. 2022;36:e37873.

\begin{abstract}
Objetivo: analisar a temática das publicações referentes à atuação das enfermeiras em pandemias. Método: revisão integrativa da literatura em base de dados da Biblioteca Virtual em Saúde, no período de maio a junho de 2020. Utilizou-se a técnica de análise temática de conteúdo para a análise dos dados. Resultados: a amostra final foi composta de 15 artigos para avaliação. Os achados foram categorizados nos temas: riscos ocupacionais, gestão, condições de trabalho e educação em saúde. Percebeu-se que a macro categoria trabalho está dissociada do reconhecimento, da valorização social, do desconhecimento da profissão e do processo de trabalho. Conclusão: as publicações analisadas não trazem aspectos inovadores no campo do cuidado humano e da concepção de saúde, muito menos de uma concepção de saúde que não seja a ausência de doença, além de um corpo físico, e a atuação das enfermeiras nas pandemias está relacionada à centralização do trabalho.
\end{abstract}

Descritores: Enfermagem. Enfermeiras. Pandemias.

Objective: to analyze the theme of publications related to nurses' work during pandemics. Method: integrative literature review at the Virtual Health Library database, from May to June 2020. The thematic content analysis technique was used for data analysis. Results: the final sample consisted of 15 articles for evaluation. The findings were categorized into the themes: occupational risks, management, working conditions and health education. The macro category work is dissociated from recognition, social valorization, unawareness of the profession and the work process. Conclusion: the analyzed publications do not bring innovative aspects in the field of human care and health conception, much less a conception of health other than the absence of disease, beyond a physical body, being nurses' role in the pandemics related to the work centralization.

Descriptors: Nursing. Nurses. Pandemics.

\footnotetext{
Enfermeiro. Doutor em Enfermagem. Professor Adjunto da Universidade Estadual de Feira de Santana. Feira de Santana, Bahia, Brasil. dbalmeida@uefs.br. https://orcid.org/0000-0002-231 I-6204.

Enfermeira. Universidade Federal da Bahia. Salvador, Bahia, Brasil. https://orcid.org/0000-0003-4233-9208.

Enfermeira. Universidade Estadual de Feira de Santana. Feira de Santana, Bahia, Brasil. https://orcid.org/0000-0002-0231-2855.

Enfermeira. Universidade Federal da Bahia. Salvador, Bahia, Brasil. https://orcid.org/0000-0002-I 100-933X

Cirurgião-dentista. Universidade Estadual de Feira de Santana. Feira de Santana, Bahia, Brasil. https://orcid.org/0000-0002-8396-7385

Graduando em Enfermagem. Universidade Estadual de Feira de Santana. Feira de Santana, Bahia, Brasil. https://orcid.org/0000-0003-4803-7737.
} 
Objetivo: analizar el tema de las publicaciones relacionadas con el trabajo de las enfermeras en pandemias. Método: revisión integradora de la literatura en la base de datos de la Biblioteca Virtual de Salud, de mayo a junio de 2020. La técnica de análisis de contenido temático se utilizó para el análisis de datos. Resultados: la muestra final consistió en 15 artículos para su evaluación. Los hallazgos se clasificaron en los temas: riesgos laborales, gestión, condiciones de trabajo y educación sanitaria. Se observó que la categoría macro trabajo está desvinculada del reconocimiento, la valorización social, el desconocimiento de la profesión y del proceso de trabajo. Conclusión: las publicaciones analizadas no aportan aspectos innovadores en el campo de la atención bumana y la concepción de la salud, y mucho menos una concepción de la salud distinta de la ausencia de enfermedad, además de un cuerpo físico, y el papel de las enfermeras en las pandemias está relacionado con la centralización del trabajo.

Descriptores: Enfermería. Enfermeras. Pandemias.

\section{Introdução}

A Enfermagem é uma ação ou uma atividade realizada predominantemente por mulheres que precisam dela para reproduzir sua própria existência, utilizam de saber advindo de outras ciências e de uma síntese produzida por ela própria, para apreender seu objeto de trabalho naquilo que interessa ao campo do cuidado na profissão, objetivando atender às necessidades sociais e de saúde da população brasileira, guardando, portanto, intrínseca relação com a dimensão política e seus processos sociais ${ }^{(1)}$.

A enfermeira baseia a sua atuação na essência e na especificidade do cuidado ao indivíduo, à família ou à comunidade. Seu escopo de atribuições próprias é marcado pela indissociabilidade da assistência e gestão e exercido nas dimensões da promoção da saúde, prevenção de doenças, recuperação e reabilitação da saúde. Sendo assim, as enfermeiras têm como objeto de trabalho o cuidado à pessoa, por meio de um processo de Enfermagem que deve ser construído mediante teorias específicas, visando o conforto, o acolhimento e o bem-estar da pessoa ${ }^{(2)}$.

De modo mais específico, as dimensões do conhecimento da Enfermagem são: empírica, ética, pessoal, estética e conhecimento emancipatório. A empírica é baseada em conhecimentos populares. A ética refere-se a valores sociais e comportamentais que podem estar vinculados ao código profissional. A pessoal significa o autoconhecimento e a autorrealização, os quais relacionam-se horizontalmente. A estética é relacionada às questões de educação em saúde, ensinar e aprender. Já o conhecimento emancipatório é contextualizado mediante a análise crítica da situação e do ambiente em que ocorre a experiência de ensino e aprendizagem ${ }^{(3)}$.

Quanto aos campos de atuação da enfermeira e o seu processo de trabalho, identificam-se os seguintes: administrar, assistir, ensinar, pesquisar e participar politicamente. Estes são dinâmicos e apresentam oportunidades constantes, estão contidos um no outro, apresentando seus elementos e a inter-relação entre eles, demonstrando a sua complexidade e que o trabalho da Enfermagem é multifacetado, requer um conjunto de conhecimentos, habilidades e atitudes que se articulam de maneira própria no cotidiano dos serviços e nos sistemas de saúde; esse processo de trabalho precisou adaptar-se à nova realidade pandêmica ${ }^{(4)}$.

Nesse cenário, o Brasil registrou o primeiro caso confirmado da COVID-19 em 26 de fevereiro de 2020, importado da Itália ${ }^{(5)}$, e até o dia 20 de abril de 2020, já haviam sido registrados 40.581 casos confirmados e 2.845 óbitos no país ${ }^{(6)}$, desencadeando a necessidade de uma série de ações estratégicas para conter a disseminação e o número de hospitalizações. Sob esse prisma, os profissionais de saúde, especialmente enfermeiras e médicos, têm sido apontados como os atores do front da pandemia, pois têm desempenhado importante papel nas dimensões de gestão, pesquisa e assistência, estando recorrentemente presentes nas discussões e notícias relativas à COVID-19 ${ }^{(7)}$.

Sendo assim, no contexto das equipes de saúde, a Enfermagem constitui mais da metade da força de trabalho no Brasil e emerge a necessidade de reinventar e valorizar a profissão, 
por meio da qualificação e desenvolvimento dos profissionais alinhados ao Sistema Único de Saúde (SUS). Aposta-se no fortalecimento da liderança da enfermagem, que mesmo com papel marcante no enfrentamento da pandemia, carece de protagonismo político e de gestão para a tomada de decisão e conquista de direitos ${ }^{(8)}$.

Paralelo a esses apontamentos, ao pensar na centralidade do trabalho da enfermeira, em especial, em situações de crise, epidemias e pandemias, e ao mesmo tempo, quando analisamos a problemática social desse campo, marcado por uma divisão social e técnica do trabalho, este artigo questiona: como é construída no campo científico a atuação das enfermeiras em tempos de pandemia? Esta pesquisa tem como objetivo analisar a temática das publicações brasileiras referentes à atuação das enfermeiras em tempo de pandemia.

\section{Método}

Esta pesquisa utilizou dois métodos de estudo, um para obtenção e o outro para análise dos dados. Na primeira etapa, para a obtenção dos dados, realizou-se revisão integrativa da literatura cuja finalidade foi sintetizar resultados obtidos por pesquisas sobre um tema ou questão, de forma abrangente, ordenada e sistemática $^{(9-10)}$. Fundamentou-se na constituição de uma análise mais vasta da literatura, fato que colabora para reflexões referentes aos métodos e resultados de pesquisa e possibilita discussões sobre a realização de futuras investigações ${ }^{(11)}$. Posteriormente, realizou-se a análise temática de conteúdo, que se caracteriza como uma técnica para análise de informações sobre o comportamento humano, possibilitando uma aplicação bastante variada, e tem duas funções: verificação de hipóteses e/ou questões e descoberta dos conteúdos manifestos ${ }^{(12)}$.

Para a revisão da literatura, foram seguidos os passos recomendados em estudo ${ }^{(9)}$, que indica como etapa inicial a identificação do tema e a seleção da hipótese ou pergunta, que, para este estudo, foi a seguinte: "Como é construída no campo científico brasileiro a atuação das enfermeiras em tempos de pandemia?” Em seguida, avançou-se para as demais etapas, na seguinte ordem: definição de critérios de inclusão e exclusão, seleção da amostra, determinação das informações a serem coletadas e categorização dos estudos selecionados, análise e discussão dos achados até atingir a síntese do conhecimento.

Para cumprimento dessa etapa, o levantamento bibliográfico foi realizado mediante consulta à base de dados da Biblioteca Virtual em Saúde (BVS), no período de maio a junho de 2020. A estratégia de busca utilizada foi composta pelos seguintes descritores combinados: (enfermagem OR nursing OR enfermería) AND (enfermeira OR enfermeiras OR nurse OR nurses OR enfermera) AND (enfermeiro OR enfermeiros) AND (pandemias OR pandemic OR pandemia). Os critérios de inclusão adotados foram: artigos originais ou relatos de experiência nos idiomas português, inglês e espanhol relacionados à temática, de qualquer ano e com disponibilidade de acesso gratuito. Os critérios de exclusão utilizados foram artigos incompletos ou que não abordavam a temática proposta. Após a utilização da estratégia de busca, foram encontrados 49 artigos, e após a aplicação dos critérios apenas 15 seguiram para análise.

Após a seleção dos artigos, seguiu-se com a determinação das informações relevantes para a pesquisa, categorização, análise e sumarização dos resultados. Para se alcançar uma organização eficiente, os achados foram categorizados em um quadro-síntese, nos temas riscos ocupacionais, gestão, condições de trabalho e educação em saúde ${ }^{(10-11)}$, e posteriormente avaliados mediante a técnica de análise temática de conteúdo.

Destaca-se que não foi necessário submeter esta pesquisa ao Comitê de Ética em Pesquisa por se tratar de estudo que utiliza dados secundários oriundos de outros estudos ${ }^{(13)}$.

\section{Resultados}

Os resultados desta pesquisa estão apresentados nos dois quadros seguintes. O Quadro 1 descreve o título, tipo de estudo, objetivos e categorias dos artigos. 
Quadro 1 - Caracterização dos estudos incluídos na revisão integrativa segundo título do artigo, tipo de estudo, objetivo e categorias. Brasil, maio-jun. 2020

\begin{tabular}{|c|c|c|c|}
\hline Título do artigo & Tipo de estudo & Objetivo(s) & Categoria \\
\hline $\begin{array}{l}\text { Expanding nursing's role } \\
\text { in responding to global } \\
\text { pandemics } 5 / 14 / 2018^{(14)}\end{array}$ & Qualitativo & $\begin{array}{l}\text { Analisar e reconhecer os } \\
\text { principais sintomas da doença, } \\
\text { visando alertar a população e } \\
\text { os serviços preventivos para } \\
\text { impedir e/ou reduzir a difusão } \\
\text { do vírus. }\end{array}$ & $\begin{array}{l}\text { Educação em } \\
\text { saúde }\end{array}$ \\
\hline $\begin{array}{l}\text { Moral Distress in the } \\
\text { Midst of the COVID-19 } \\
\text { Pandemic }{ }^{(15)} \text {. }\end{array}$ & Qualitativo & $\begin{array}{l}\text { Analisar o sofrimento moral } \\
\text { dos enfermeiros durante a } \\
\text { pandemia da COVID-19. }\end{array}$ & Risco ocupacional \\
\hline $\begin{array}{l}\text { COVID-19 in the Year of } \\
\text { the Nurse }{ }^{(16)} \text {. }\end{array}$ & Qualitativo & $\begin{array}{l}\text { Analisar o crescente } \\
\text { adoecimento dos enfermeiros } \\
\text { frente a essa pandemia. }\end{array}$ & Risco ocupacional \\
\hline $\begin{array}{l}\text { A qualitative study } \\
\text { on the psychological } \\
\text { experience of caregivers } \\
\text { of COVID-19 patients }^{(17)} \text {. }\end{array}$ & Qualitativo & $\begin{array}{l}\text { Explorar a psicologia de } \\
\text { enfermeiros que cuidam de } \\
\text { pacientes com COVID-19. }\end{array}$ & Risco ocupacional \\
\hline $\begin{array}{l}\text { Psychological stress of } \\
\text { ICU nurses in the time of } \\
\text { COVID-19 }\end{array}$ & Qualitativo & $\begin{array}{l}\text { Avaliar os impactos psicológicos } \\
\text { que os enfermeiros recém- } \\
\text {-formados sofrem durante a } \\
\text { infecção por COVID-19. }\end{array}$ & Risco ocupacional \\
\hline $\begin{array}{l}\text { Los valores profesionales } \\
\text { de las enfermeras(os) } \\
\text { chilenas(os) en tiempos } \\
\text { de crisis sanitaria por } \\
\text { Covid-19 } 9^{(19)} \text {. }\end{array}$ & Qualitativo & $\begin{array}{l}\text { Analisar os valores e os } \\
\text { comportamento de uma } \\
\text { pessoa e dos enfermeiros } \\
\text { frente à pandemia da } \\
\text { Covid-19. }\end{array}$ & Risco ocupacional \\
\hline $\begin{array}{l}\text { Gestão da pandemia } \\
\text { coronavírus em um } \\
\text { hospital: relato de } \\
\text { experiência } \\
\text { professional }^{(20)}\end{array}$ & Qualitativo & $\begin{array}{l}\text { Descrever a experiência da } \\
\text { gestão para o atendimento } \\
\text { de paciente confirmado ou } \\
\text { com suspeita de Coronavírus } \\
\text { em um hospital da região } \\
\text { metropolitana de Porto Alegre. }\end{array}$ & Gestão \\
\hline $\begin{array}{l}\text { Gestión y liderazgo } \\
\text { de los servicios de } \\
\text { enfermería en el plan } \\
\text { de emergencia de la } \\
\text { pandemia COVID-19: La } \\
\text { experiencia del Hospital } \\
\text { Clínic de Barcelona }^{(21)} \text {. }\end{array}$ & Qualitativo & $\begin{array}{l}\text { Analisar as demandas dos } \\
\text { enfermeiros para gerenciar } \\
\text { a pandemia da COVID-19, } \\
\text { além de colocar um desafio } \\
\text { tanto na provisão de pessoal } \\
\text { quanto em suprimentos e } \\
\text { material sanitário. }\end{array}$ & Gestão \\
\hline $\begin{array}{l}\text { What the COVID-19 } \\
\text { pandemic tells us about } \\
\text { the need to develop } \\
\text { resilience in the nursing } \\
\text { workforce }^{(22)}\end{array}$ & Qualitativo & $\begin{array}{l}\text { Analisar brevemente as } \\
\text { pandemias e os desastres } \\
\text { anteriores que afetaram os } \\
\text { sistemas de saúde, bem } \\
\text { como a pandemia } 2020 \\
\text { COVID-19, e considera como } \\
\text { as enfermeiras líderes podem } \\
\text { apoiar a equipe e mostrar } \\
\text { resiliência organizacional } \\
\text { durante essa emergência. }\end{array}$ & Gestão \\
\hline
\end{tabular}


Quadro 1 - Caracterização dos estudos incluídos na revisão integrativa segundo título do artigo, tipo de estudo, objetivo e categorias. Brasil, maio-jun. 2020

\begin{tabular}{|c|c|c|c|}
\hline Título do artigo & Tipo de estudo & Objetivo(s) & Categoria \\
\hline $\begin{array}{l}\text { Estimating Weekly Call } \\
\text { Volume to a National } \\
\text { Nurse Telephone Triage } \\
\text { Line in an Influenza } \\
\text { Pandemic }{ }^{(23)} \text {. }\end{array}$ & Quantitativa & $\begin{array}{l}\text { Analisar as linhas de triagem } \\
\text { de Enfermagem e as taxas de } \\
\text { chamada para hospitalização } \\
\text { com base na experiência } \\
\text { com a linha de triagem de } \\
\text { enfermagem. }\end{array}$ & Gestão \\
\hline $\begin{array}{l}\text { Influenza infection } \\
\text { control practices in labor } \\
\text { and delivery units during } \\
\text { the } 2009 \mathrm{H}^{(1 N 1} \text { influenza } \\
\text { pandemic }{ }^{(24)} \text {. }\end{array}$ & Qualitativo & $\begin{array}{l}\text { Avaliar a presença e a } \\
\text { utilidade de políticas e } \\
\text { práticas escritas sobre controle } \\
\text { de infecções, consistentes } \\
\text { com as orientações do Centro } \\
\text { de Controle e Prevenção de } \\
\text { Doenças (CDC) nas unidades } \\
\text { de trabalho e parto (L\&D) do } \\
\text { hospital durante a pandemia } \\
\text { de influenza H1N1 de } 2009 .\end{array}$ & Gestão \\
\hline $\begin{array}{l}\text { Nurses: Courageous, } \\
\text { Committed, and Fed Up }{ }^{(25)} \text {. }\end{array}$ & Qualitativo & $\begin{array}{l}\text { Reconhecer o papel crítico } \\
\text { que as enfermeiras e parteiras } \\
\text { desempenham na garantia da } \\
\text { saúde da população mundial. }\end{array}$ & $\begin{array}{l}\text { Condições de } \\
\text { trabalho }\end{array}$ \\
\hline $\begin{array}{l}\text { Legal aspects of } \\
\text { COVID-19 pandemic } \\
\text { management for } \\
\text { community nurses }^{(26)} \text {. }\end{array}$ & Qualitativo & $\begin{array}{l}\text { Analisar como as condições } \\
\text { de trabalho podem se } \\
\text { desenvolver em relação à } \\
\text { lei sobre responsabilidade } \\
\text { profissional e analisar } \\
\text { brevemente o provável } \\
\text { aumento de trabalhadores } \\
\text { voluntários, bem como } \\
\text { um aspecto importante da } \\
\text { confidencialidade do paciente. }\end{array}$ & $\begin{array}{l}\text { Condições de } \\
\text { trabalho }\end{array}$ \\
\hline $\begin{array}{l}100 \text { years on: the Spanish } \\
\text { Flu, pandemics and } \\
\text { keeping nurses safe }{ }^{(27)} \text {. }\end{array}$ & Qualitativo & $\begin{array}{l}\text { Analisar o contexto em que } \\
\text { as enfermeiras se encontram } \\
\text { durante as pandemias dos } \\
\text { últimos } 100 \text { anos, trazendo } \\
\text { informações sobre a } \\
\text { proporção de mortes entre } \\
\text { a população em geral e os } \\
\text { profissionais de saúde. }\end{array}$ & $\begin{array}{l}\text { Condições de } \\
\text { trabalho }\end{array}$ \\
\hline $\begin{array}{l}\text { Enfermeras y practicantes } \\
\text { durante la epidemia de } \\
\text { gripe de 1918: Análisis a } \\
\text { través de la prensa } \\
\text { Española }^{(28)} \text {. }\end{array}$ & Qualitativo & $\begin{array}{l}\text { Abordar os eventos } \\
\text { relacionados à epidemia na } \\
\text { relação com os profissionais } \\
\text { de Enfermagem desde a } \\
\text { perspectiva periódica. }\end{array}$ & $\begin{array}{l}\text { Condições de } \\
\text { trabalho }\end{array}$ \\
\hline
\end{tabular}

Fonte: Elaboração própria.

O Quadro 2 descreve os principais resultados dos artigos analisados segundo título do artigo e os resultados encontrados. 
Quadro 2 - Descrição dos principais resultados dos artigos selecionados segundo título do manuscrito e seus resultados. Brasil, maio-jun. 2020

(continua)

\begin{tabular}{|c|c|}
\hline Artigo & Resultados \\
\hline $\begin{array}{l}\text { Psychological stress of ICU nurses in the } \\
\text { time of COVID-19 }\end{array}$ & $\begin{array}{l}\text { Devido ao aumento do número de casos da pandemia } \\
\text { pela COVID-19, os sistemas de saúde se sobrecarregam } \\
\text { e geram sofrimento psíquico aos enfermeiros. Ao } \\
\text { pesquisar } 85 \text { enfermeiros da UTI, percebeu-se que as } \\
\text { principais manifestações foram: diminuição do apetite } \\
\text { ou indigestão (59\%), fadiga ( } 55 \%) \text {, dificuldade para } \\
\text { dormir ( } 45 \%) \text {, nervosismo ( } 28 \%) \text {, choro frequente } \\
\text { (26\%), e até pensamentos suicidas ( } 2 \%) \text {. Nota-se } \\
\text { ainda que os enfermeiros sem experiência profissional } \\
\text { enfrentam crise psicológica maior. Logo, estes impactos } \\
\text { psicológicos devem ser avaliados e resolvidos, para que } \\
\text { não se torne fator de risco de infecção por COVID-19, } \\
\text { visto que este fator influencia na imunidade e na } \\
\text { autoproteção, como também na qualidade e segurança } \\
\text { do sistema de saúde. }\end{array}$ \\
\hline $\begin{array}{l}\text { Moral Distress in the Midst of the } \\
\text { COVID-19 Pandemic }{ }^{(15)} \text {. }\end{array}$ & $\begin{array}{l}\text { Os enfermeiros na linha de frente do tratamento de } \\
\text { pacientes com COVID-19 correm risco em vários níveis } \\
\text { de desenvolver sofrimento moral. A comunidade pode } \\
\text { ajudar a apoiar os enfermeiros ficando em casa, para } \\
\text { achatar a curva, e mantendo distância social quando } \\
\text { precisar sair para obter suprimentos essenciais. Além } \\
\text { disso, pode-se fazer atos aleatórios de bondade pelos } \\
\text { enfermeiros em nossas vidas. }\end{array}$ \\
\hline COVID-19 in the Year of the Nurse ${ }^{(16)}$ & $\begin{array}{l}\text { Os enfermeiros estão se tornando pacientes devido à } \\
\text { nova pandemia que assola o mundo, a COVID-19. Isso } \\
\text { está acontecendo por falta de conhecimento sobre } \\
\text { essa nova pandemia ou pela escassez de recursos } \\
\text { necessários para combatê-la com segurança. Além } \\
\text { disso, informa sobre os sinais e sintomas da COVID-19, } \\
\text { a qual tem início rápido, causando aumento nas } \\
\text { internações e na capacidade dos sistemas de saúde, } \\
\text { pois se não forem adequadamente abordados, } \\
\text { comprometem os pacientes e a equipe do hospital que } \\
\text { presta os cuidados. }\end{array}$ \\
\hline $\begin{array}{l}\text { A qualitative study on the psychological } \\
\text { experience of caregivers of COVID-19 } \\
\text { patients }{ }^{(17)} \text {. }\end{array}$ & $\begin{array}{l}\text { A experiência psicológica de enfermeiros que cuidam } \\
\text { de pacientes com COVID-19 pode ser resumida } \\
\text { em quatro temas. Primeiro, as emoções negativas } \\
\text { presentes no estágio inicial, que consistem em fadiga, } \\
\text { desconforto e desamparo, foram causadas por trabalho } \\
\text { de alta intensidade, medo, ansiedade e preocupação } \\
\text { com os pacientes e familiares. Segundo, os estilos de } \\
\text { autocontrole incluíam ajuste psicológico e de vida, } \\
\text { atos altruístas, apoio à equipe e cognição racional. } \\
\text { Terceiro, crescimento sob pressão, que incluía maior } \\
\text { afeto e gratidão, desenvolvimento de responsabilidade } \\
\text { profissional e autorreflexão. Finalmente, mostrou-se } \\
\text { que as emoções positivas ocorreram simultaneamente } \\
\text { com emoções negativas. }\end{array}$ \\
\hline
\end{tabular}


Quadro 2-Descrição dos principais resultados dos artigos selecionados segundo título do manuscrito e seus resultados. Brasil, maio-jun. 2020

(continuação)

\begin{tabular}{|c|c|}
\hline Artigo & Resultados \\
\hline $\begin{array}{l}\text { Los valores profesionales de las } \\
\text { enfermeras(os) chilenas(os) en tiempos de } \\
\text { crisis sanitaria por Covid-19 }\end{array}$ & $\begin{array}{l}\text { Os enfermeiros no Chile enfrentam novas situações } \\
\text { médicas desconhecidas e que podem ameaçar suas vidas. } \\
\text { Os estudantes e colegas observam como os valores } \\
\text { profissionais, tão intimamente ligados ao profissional, os } \\
\text { fortalecem, promovendo suas identidades profissionais } \\
\text { e os comportamentos de trabalho adequados, como } \\
\text { priorizar as necessidades e as demandas de atendimento } \\
\text { de indivíduos, famílias e comunidades, cuidar deles, } \\
\text { proteger seus direitos morais e legais, fornecer cuidados } \\
\text { dedicados, humanos e de alto valor técnico, mantendo } \\
\text { sua privacidade e confidencialidade, fazendo turnos de } \\
24 \text { horas com qualidade e isolando-se de suas famílias } \\
\text { para "cuidar deles". }\end{array}$ \\
\hline $\begin{array}{l}\text { What the COVID-19 pandemic tells us } \\
\text { about the need to develop resilience in } \\
\text { the nursing workforce }{ }^{(22)} \text {. }\end{array}$ & $\begin{array}{l}\text { A maioria das pesquisas sobre resiliência em sistemas } \\
\text { de saúde, como o Sistema Nacional de Saúde, é } \\
\text { baseada em crises organizacionais, como falta de } \\
\text { enfermeiros, envelhecimento da força de trabalho e } \\
\text { restrições financeiras. No entanto, a Enfermagem pode } \\
\text { aprender lições do passado para tornar-se mais resiliente, } \\
\text { principalmente considerando a pandemia } 2020 \text { COVID-19. } \\
\text { Este artigo analisa brevemente as pandemias e os } \\
\text { desastres anteriores que afetaram os sistemas de saúde, } \\
\text { bem como a pandemia } 2020 \text { COVID-19, e considera como } \\
\text { as enfermeiras líderes podem apoiar a equipe e mostrar } \\
\text { resiliência organizacional durante essas emergências. O } \\
\text { artigo também discute como as enfermeiras líderes podem } \\
\text { desenvolver sua própria resiliência. }\end{array}$ \\
\hline $\begin{array}{l}\text { Gestão da pandemia coronavírus em um } \\
\text { hospital: relato de experiência } \\
\text { profissional }^{(20)} \text {. }\end{array}$ & $\begin{array}{l}\text { Para os enfermeiros, técnicos de Enfermagem e } \\
\text { médicos, a pandemia representa um desafio para } \\
\text { a saúde mental. Devido à grande pressão, estes } \\
\text { profissionais de saúde tendem a não cuidar da própria } \\
\text { saúde mental no constante e incansável enfrentamento } \\
\text { à COVID-19, fato que proporciona o aparecimento de } \\
\text { sofrimentos relacionados à ansiedade e ao estresse. }\end{array}$ \\
\hline $\begin{array}{l}\text { Gestión y liderazgo de los servicios de } \\
\text { enfermería en el plan de emergencia de la } \\
\text { pandemia COVID-19: la experiencia del } \\
\text { Hospital Clínic de Barcelona }^{(21)} \text {. }\end{array}$ & $\begin{array}{l}\text { O recente aparecimento da COVID-19 requer uma } \\
\text { compreensão da experiência da doença, sua análise } \\
\text { de transmissão, gravidade, isolamento e gestão dos } \\
\text { cuidados realizados. A pandemia da COVID-19 } \\
\text { colocou o sistema de saúde, a atitude e a colaboração } \\
\text { à prova do cidadão, mas, acima de tudo, o grande } \\
\text { profissionalismo de todos possibilita o atendimento } \\
\text { diário ao paciente. Os departamentos de Enfermagem } \\
\text { desempenham papel fundamental na prestação de } \\
\text { cuidados essenciais às comunidades durante esses } \\
\text { tipos de desastres, que podem levar ao rápido } \\
\text { aumento de serviços e sobrecarregar a capacidade } \\
\text { funcional e a segurança dos hospitais e do sistema } \\
\text { de saúde em geral. A capacidade de trabalhar em } \\
\text { equipe, o gerenciamento emocional e o respeito pelas } \\
\text { decisões organizacionais tomadas possibilitam que se } \\
\text { enfrente os desafios da pandemia. }\end{array}$ \\
\hline
\end{tabular}


Quadro 2 - Descrição dos principais resultados dos artigos selecionados segundo título do manuscrito e seus resultados. Brasil, maio-jun. 2020

\begin{tabular}{|c|c|}
\hline Artigo & Resultados \\
\hline $\begin{array}{l}\text { Estimating Weekly Call Volume to a } \\
\text { National Nurse Telephone Triage Line in } \\
\text { an Influenza Pandemic }{ }^{(23)} \text {. }\end{array}$ & $\begin{array}{l}\text { As linhas telefônicas de triagem de enfermeiras, como } \\
\text { a Flu on Call@ dos Centros de Controle e Prevenção } \\
\text { de Doenças (CDC), uma linha nacional de triagem } \\
\text { de enfermeiras, podem ajudar a reduzir o aumento } \\
\text { da demanda por cuidados de saúde durante uma } \\
\text { pandemia de influenza, mediante a triagem de } \\
\text { pacientes, fornecendo conselhos sobre tratamentos } \\
\text { clínicos, cuidados e informações sobre a pandemia e } \\
\text { acesso a medicamentos antivirais prescritos. }\end{array}$ \\
\hline $\begin{array}{l}\text { Influenza infection control practices in } \\
\text { labor and delivery units during the } 2009 \\
\text { H1N1 influenza pandemic }{ }^{(24)} \text {. }\end{array}$ & $\begin{array}{l}\text { Os entrevistados }(73,8 \%) \text { relataram que a orientação } \\
\text { do CDC foi muito útil para o controle de infecções } \\
\text { em locais de pesquisa e desenvolvimento durante } \\
\text { a pandemia. Avaliou-se a presença das políticas } \\
\text { escritas de controle de infecção, consistentes com } \\
\text { as orientações do CDC nas unidades hospitalares de } \\
\text { pesquisa e desenvolvimento, durante a pandemia de } \\
\text { influenza H1N1 de } 2009 \text { e sua taxa de implementação, } \\
\text { na maioria das vezes, questionando as mulheres na } \\
\text { chegada sobre sintomas recentes semelhantes à gripe } \\
\text { ( } 89,4 \%, 89,9 \%) \text {, iniciando de imediato medicamentos } \\
\text { antivirais em casos de suspeita ou confirmação } \\
\text { de gripe (65,2\%, 49\%), isolando imediatamente as } \\
\text { mulheres doentes de mulheres saudáveis ( } 90,7 \% \text {, } \\
84,7 \%) \text {, solicitando que as mulheres doentes usassem } \\
\text { máscaras durante a pesquisa e desenvolvimento (67\%, } \\
57,7 \% \text { ), separando imediatamente recém-nascidos } \\
\text { saudáveis de mães doentes ( } 50,9 \%, 42,4 \%) \text { e banhando } \\
\text { bebês saudáveis quando estáveis ( } 58,4 \%, 56,9 \%) \text {. As } \\
\text { políticas escritas relatadas para cinco das seis práticas } \\
\text { aumentaram durante a pandemia. Cinco das seis } \\
\text { políticas escritas permaneceram acima da linha de base } \\
\text { após a pandemia. }\end{array}$ \\
\hline $\begin{array}{l}\text { Nurses: Courageous, Committed, } \\
\text { and Fed Up }\end{array}$ & $\begin{array}{l}\text { As enfermeiras estão se afastando e se manifestando. } \\
\text { A Associação de Enfermeiras do Estado de Nova } \\
\text { York entrou com uma ação contra o Estado e vários } \\
\text { hospitais por falha na proteção dos trabalhadores. Os } \\
\text { hospitais estão lutando contra uma provisão no projeto } \\
\text { de combate da COVID-19 que estabeleceria padrões } \\
\text { para garantir a proteção dos enfermeiros. Vários outros } \\
\text { sindicatos estão se unindo a esses esforços, observando, } \\
\text { em uma declaração conjunta, que os enfermeiros não } \\
\text { estão dispostos a perder desnecessariamente suas vidas, } \\
\text { deixando seus pacientes e familiares para trás, apenas } \\
\text { porque os empregadores e o governo não investem no } \\
\text { mais alto nível de proteção. }\end{array}$ \\
\hline $\begin{array}{l}\text { Legal aspects of COVID-19 pandemic } \\
\text { management for community nurses }{ }^{(26)} \text {. }\end{array}$ & $\begin{array}{l}\text { Entre as maiores preocupações referente ao } \\
\text { atendimento dos pacientes, destaca-se a assistência } \\
\text { prestada pelos profissionais que contraíram o vírus, } \\
\text { visto que já houveram relatos de casos em que os } \\
\text { profissionais foram o meio de transmissão. Os médicos } \\
\text { aparecem em destaque no que se refere a esses casos, } \\
\text { e até o momento, existem poucos relatos do efeito } \\
\text { dessa situação perante os enfermeiros. Entretanto, a } \\
\text { probabilidade é que comece a existir. }\end{array}$ \\
\hline
\end{tabular}


Quadro 2-Descrição dos principais resultados dos artigos selecionados segundo título do manuscrito e seus resultados. Brasil, maio-jun. 2020

(conclusão)

\begin{tabular}{|l|l|}
\hline Artigo & \multicolumn{1}{c|}{ Resultados } \\
\hline $\begin{array}{l}100 \text { years on: the Spanish Flu, pandemics } \\
\text { and keeping nurses safe }{ }^{(27)} .\end{array}$ & $\begin{array}{l}\text { São necessários investimentos na força de trabalho em } \\
\text { saúde para cumprir o compromisso com a Cobertura } \\
\text { Universal de Saúde e a crescente demanda por } \\
\text { cuidados qualificados para pessoas com condiçoses } \\
\text { complexas e necessidades de saúde. Os enfermeiros } \\
\text { devem estar no centro do palco para atender a essas } \\
\text { necessidades e estar adequadamente equipados e } \\
\text { apoiados. O estudo da Lancet também concluiu que } \\
\text { investimentos substanciais nos sistemas de saúde - e } \\
\text { especificamente na força de trabalho em saúde - são } \\
\text { urgentemente necessários, não apenas para melhorar } \\
\text { a preparação para futuras epidemias e atender às } \\
\text { necessidades básicas, mas também para limitar os } \\
\text { efeitos secundários à saúde da epidemia de Ebola. }\end{array}$ \\
\hline $\begin{array}{l}\text { Enfermeras y practicantes durante la } \\
\text { epidemia de gripe de 1918: Ańálisis a } \\
\text { través de la prensa española }{ }^{(28)} .\end{array}$ & $\begin{array}{l}\text { Há escassez de informações em termos quantitativo } \\
\text { sobre os profissionais de Enfermagem, os cuidados } \\
\text { que eles ofereceram e as tarefas que executaram, } \\
\text { caracterizando uma situação que poderia ser rotulada de } \\
\text { invisibilidade e subordinação que perdurou ao longo do } \\
\text { século XX e que ainda continua atual, o que influenciou } \\
\text { socialmente no baixo reconhecimento da profissão. }\end{array}$ \\
\hline $\begin{array}{l}\text { Expanding nursing's role in responding to } \\
\text { global pandemics } 5 / 14 / 2018^{(29)}\end{array}$ & $\begin{array}{l}\text { Uma ameaça para as possíveis pandemias é o atraso na } \\
\text { identificação antecipada de infecções. Para reduzir esse } \\
\text { fato, é necessário que o profissional de saúde propicie } \\
\text { uma comunicação eficiente com a pessoa infectada, } \\
\text { para que possa iniciar a identificação do patógeno, } \\
\text { O tratamento adequado e a prevenção de maior } \\
\text { circulação viral. Para além disso, o reconhecimento } \\
\text { dos principais sintomas, visando alertar a população } \\
\text { e os serviços preventivos para impedir e/ou reduzir a } \\
\text { difusão do vírus. }\end{array}$ \\
\hline
\end{tabular}

Fonte: Elaboração própria.

\section{Discussão}

A revisão realizada neste estudo demonstrou que apenas 1 artigo selecionado para avaliação tratou de pesquisa quantitativa, enquanto que 14 artigos foram escritos na perspectiva qualitativa. Quanto à categoria desses estudos, foi constatado que dos 15 selecionados, 5 tratava-se de risco ocupacional, 5 de gestão, 4 de condições de trabalho e apenas 1 discutia aspectos da educação em saúde. Sendo assim, ficou constatado que a atuação da enfermeira abrange diversos campos, entre os quais podemos destacar: gestão de serviços e sistemas de saúde, cuidado direto aos indivíduos e coletividades (saudáveis ou não), promoção e prevenção da saúde, educação popular em saúde, ensino e a formação em saúde e em enfermagem, bem como atividades de pesquisa e extensão ${ }^{(29)}$.

Os estudos categorizados versam sobremaneira na temática dos riscos ocupacionais e estão relacionados com as condições de trabalho em Enfermagem e Saúde, em especial em pandemias, representando a maioria dos estudos identificados. Entre os riscos ocupacionais, nota-se o sofrimento moral, sendo destacáveis os sentimentos e vivências de raiva, ansiedade, frustração, insônia, e até mesmo pensamentos suicidas, que são aumentados pela sensação de incapacidade em tomar atitudes para lidar com o 
sofrimento, visto que esse estado pode ser predisposto por situações internas e individuais, externas e/ou devido a posturas institucionais ${ }^{(15-17)}$.

Contextualizando os riscos ocupacionais, evidencia-se o crescente adoecimento dos enfermeiros frente às pandemias. Fato que demostra a importância da segurança da Saúde nos serviços, por meio de acesso a condições de trabalho adequadas, bem como as questões específicas estruturais, vinculadas a modelos de atenção e gestão, a cultura organizacional, a disponibilidade de tecnologias do cuidado e aos equipamentos de proteção individual (EPIs), dimensionamento de pessoal adequado às prerrogativas legais, éticas e técnicas, serviços de atendimento às famílias das enfermeiras, treinamento e interação interpessoal para facilitar a adaptação e o suporte das enfermeiras no enfrentamento às pandemias ${ }^{(18)}$.

Nessa perspectiva, outros estudos destacam que as enfermeiras identificam que o enfrentamento das pandemias acirra a incidência de riscos ocupacionais, e isto gera apreensão, como por exemplo: maior probabilidade de infecção, sobrecarga de trabalho, preocupação com os familiares que podem se infectar por ter contato consigo e aumento no nível de estresse no trabalho. Torna-se imprescindível considerar esses aspectos no plano e na prática de contingência locorregional da pandemia ${ }^{(19)}$.

Posteriormente, identificou-se a concentração de estudos na temática de gestão com relação aos cuidados, serviços de saúde e equipe. O trabalho da enfermeira tem como escopo de atuação a gestão de serviços e dos sistemas de saúde. Isso expressa a sua centralidade, pois quando se pensa na existência de outras práticas de saúde, geralmente a enfermeira articula a prestação de cuidado de todos os outros profissionais, seja na atenção básica de saúde, no componente hospitalar e ambulatorial e na reabilitação ${ }^{(20-21)}$.

Além disso, o gerenciamento do serviço pela enfermeira ocupa-se de questões vinculadas à infraestrutura, administração de recursos materiais, gerenciamento de resíduos sólidos, dimensionamento, previsão, provisão e distribuição de pessoal, gerenciamento da equipe de enfermagem e ainda a indissociabilidade com o cuidado direto aos pacientes críticos ${ }^{(22)}$. Ademais, quando se fala em pandemias no aspecto do gerenciamento, fala-se também na capacidade de gerenciar uma equipe multiprofissional, destacando o escopo das atribuições da enfermeira e a prestação de cuidado a pessoas, famílias e comunidades ${ }^{(23-24)}$.

A terceira categoria identificada na análise de dados foram as condições de trabalho, que se entrelaçam com os riscos ocupacionais; de modo mais específico, identificou-se que as questões históricas, econômicas, de gênero e religiosas da Enfermagem impactam no valor de sua força de trabalho, bem como no reconhecimento e visibilidade profissional ${ }^{(25)}$. Por outro lado, estudos apontam que profissionais de saúde e Enfermagem são identificados como alto risco e vulnerabilidade em situações de enfrentamento a uma pandemia ${ }^{(26)}$. As condições de trabalho quando já são precárias, ficam mais expressas em uma situação de crise, como a pandemia.

Os destaques são amplamente discutidos na literatura quando se trata do trabalho da enfermeira, dentre os quais extensas jornadas, baixos salários, submissão, fragilidade de identidade profissional, ritmo intenso de trabalho, desvalorização profissional, conflitos interpessoais, entre outros fatores desencadeantes de desgastes físicos e psíquicos. No momento de pandemia, estas condições são potencializadas pela crise que passa os serviços e os sistemas de saúde.

O valor da vida das enfermeiras e sua responsabilidade civil entram em conflito, podendo trazer consequências para sua saúde e segurança no desempenho de suas atividades laborais, já que, sob maior pressão, esses profissionais tendem a descuidar da própria saúde mental na incansável e constante luta contra as pandemias, podendo propiciar o surgimento de transtornos relacionados ao estresse e ansiedade ${ }^{(27)}$.

As enfermeiras estão submetidas à intensidade do trabalho, às condições de trabalho precárias e a modelos de gestão do processo de trabalho que ampliam a exploração e a exposição a questões insalubres. As dimensões da precarização analisadas afetam as trabalhadoras e também a prática clínica e gerencial, e impedem ou limitam a assistência prestada ao usuário, 
pois há um profundo distanciamento do preconizado nas condições de trabalho no cotidiano dos serviços de saúde, apesar de asseguradas no campo legal e ético ${ }^{(28)}$. Além disso, a intensidade do trabalho produzida pelo acúmulo de vínculos empregatícios, as fragilidades organizacionais e do trabalhador, potencializadas pela situação de crise, predispõem as enfermeiras à fadiga física, mental e psicológica, o que pode contribuir para a ocorrência de erros na assistência.

Vale destacar que, por outro lado, as instâncias de representação profissional e fiscalização expressam a fragilidade política da categoria e ainda precisam de maior fortalecimento no campo de disputas sociais, tanto no macro espaço como no micro espaço das relações ${ }^{(28)}$.

$\mathrm{Na}$ quarta categoria aparece um estudo sobre a temática da educação em saúde que, por sua vez, tem importância na construção de uma consciência sanitária, algo primaz em épocas de pandemia, possuindo imbricação com todas as dimensões da saúde vinculadas a práticas informativas e formativas direcionadas à população: promoção, prevenção, cura e reabilitação ${ }^{(14)}$.

Por fim, destaca-se a inexistência de estudos que tratem dos aspectos específicos do cuidado em Enfermagem, da invisibilidade das teorias e do processo de Enfermagem, que repercute em prejuízo para a população e para o campo da saúde de modo geral. Fica o cuidado da enfermeira vinculado exclusivamente a descobertas científicas médicas, como se esta fosse uma mera cumpridora de prescrição médica e não tivesse um campo autônomo para exercício profissional.

Sendo assim, destacou-se que os estudos, em sua totalidade, estão vinculados à macro categoria trabalho, com o trabalho definido como uma práxis social, com repercussão no campo individual e coletivo, de caráter social, complexo, dinâmico, mutante e que se distingue de qualquer outro tipo de prática animal por sua natureza reflexiva, dialética, consciente, propositiva, estratégica, instrumental e moral ${ }^{(30)}$.

Em termos práticos, o trabalho da enfermeira é um campo importante de estudos, principalmente em tempos de pandemia, pois repercutirá a destruição de modo mais evidente do processo de perda dos direitos sociais que vive os brasileiros, com 1.990.202 trabalhadores de Enfermagem afetados pela reestruturação do trabalho, que promoveu a precarização das relações e das condições de trabalho, acarretando na multiplicidade de vínculos, baixos salários, comprometimento dos diretos trabalhistas, sujeição às condições indignas de trabalho, impactando consequentemente no processo de trabalho, convivendo com a insegurança relacionada à perda do emprego e à ausência de seguridade social, que contribui para aceitação da exploração e submissão a condições indignas de trabalho ${ }^{(31)}$.

\section{Conclusão}

A análise dos artigos selecionados constatou a concentração das pesquisas na temática de risco ocupacional e gestão, o que demonstra que, durante as pandemias, a expressão do trabalho da enfermeira está detida no campo da gestão e a problemática do cotidiano dos serviços é potencializada com o acirramento dos riscos ocupacionais. Além disso, expressam também o desconhecimento do fazer da enfermeira, do seu processo e teorias da Enfermagem, não sendo identificado nenhum estudo que tratasse de propostas do cuidado direto ao paciente vinculados ao seu saber em específico.

Os achados evidenciam a macro categoria trabalho, trazendo os dilemas dos serviços de saúde, as disputas sociais de poder e a valorização social, bem como a precariedade dos serviços e das condições de trabalho a que estão expostas a enfermeira e sua equipe. Não obstante a centralidade do trabalho da enfermeira e o diverso campo de atuação, os estudos não trazem aspectos inovadores do campo do cuidado humano, muito menos de uma concepção de saúde que não seja a ausência da doença, além de um corpo físico. Sendo assim, refletem práticas centradas em questões biológicas e/ou gerenciais.

Por fim, é importante reconhecer a fragilidade política do campo profissional, a estruturação das associações, sindicatos e conselho para o enfrentamento das disputas em jogo quando se trata do trabalho, de quem vende e de quem 
compra a força de trabalho, sua representação e reconhecimento, e isto passa pela formação profissional e técnica, bem como pelo reconhecimento de classe social.

\section{Colaborações:}

1 - concepção, projeto, análise e interpretação dos dados: Deybson Borba de Almeida, Laiane da Silva Santana, Maria Talita Cruz Silva Oliveira, Nívia Vanessa Carneiro dos Santos, Igor Ferreira Borba de Almeida e Caio Moura dos Santos;

2 - redação do artigo e revisão crítica relevante do conteúdo intelectual: Deybson Borba de Almeida, Laiane da Silva Santana, Maria Talita Cruz Silva Oliveira, Nívia Vanessa Carneiro dos Santos, Igor Ferreira Borba de Almeida e Caio Moura dos Santos;

3 - aprovação final da versão a ser publicada: Deybson Borba de Almeida, Laiane da Silva Santana, Maria Talita Cruz Silva Oliveira, Nívia Vanessa Carneiro dos Santos, Igor Ferreira Borba de Almeida e Caio Moura dos Santos.

\section{Referências}

1. Almeida MCP, Rocha SMM. O trabalho de Enfermagem. São Paulo: Cortez, 1997.

2. Magalhães SSA, Machado CJ. Conceitos epidemiológicos e pandemias recentes: novos desafios. Cad saúde colet. 2014;22(1):109-10. DOI: https://doi.org/10.1590/1414-462X201400010016

3. Chinn PL. Critical theory and emancipatory knowing. In: Rich KL, Butts JB, editors. Philosophies and theories for advanced nursing practice. Massachusettes: Jones \& Bartlett Learning; 2015. p. 143-63.

4. Sanna MC. Os processos de trabalho em Enfermagem. Rev bras enferm. 2007 Apr;60(2):221-4. DOI: https://doi.org/10.1590/ S0034-71672007000200018

5. Croda JHR, Garcia LP. Resposta imediata da Vigilância em Saúde à epidemia da COVID-19. Epidemiol Serv Saúde. 2020;29(1):e2020002. DOI: http://dx.doi.org/10.5123/s1679-497420200001 00021

6. Brasil. Ministério da Saúde. Coronavírus Brasil. Painel Coronavírus [Internet]. Brasília (DF); 2020 [cited 2020 Apr 20]. Available from: https://COVID. saude.gov.br/

7. Moreira MRC, Xavier SPL, Machado LDS, Silva MRF, Machado MFAS. Enfermagem na pandemia da COVID-19: análise de reportagens à luz da Teoria do Reconhecimento. Enferm Foco 2020;11(1esp):116-23. DOI: https://doi. org/10.21675/2357-707X.2020.v11.n1.ESP.3581

8. Daly J, Jackson D, Anders R, Davidson PM. Who speaks for nursing? COVID-19 highlighting gaps in leadership. J Clin Nurs. 2020;29(15-16):2751-2. DOI: 10.1111/jocn.15305

9. Soares CB, Hoga LAK, Peduzzi M, Sangaleti C, Yonekura T, Silva DRAD. Integrative Review: Concepts and Methods Used in Nursing. Rev esc enferm USP. 2014;48(2):335-45. DOI: https://doi. org/10.1590/S0080-6234201400002000020

10. Pompeo DA, Rossi LA, Galvão CM. Integrative literature review: the initial step in the validation process of nursing diagnoses. Acta paul enferm. 2009;22(4):434-8. DOI: https://doi.org/10.1590/ S0103-21002009000400014

11. Mendes KDS, Silveira RCCP, Galvão CM. Revisão integrativa: método de pesquisa para a incorporação de evidências na saúde e na enfermagem. Texto contexto - enferm. 2008;17(4):758-64. DOI: http://dx.doi.org/10.1590/ S0104-07072008000400018

12. Minayo MCS (org.). Pesquisa Social: teoria, método e criatividade. 18a ed. Petrópolis (RJ): Vozes; 2001.

13. Brasil. Ministério da Saúde. Resolução n. 466, de 12 de dezembro de 2012. Aprova as diretrizes e normas regulamentadoras de pesquisas envolvendo seres humanos [Internet]. Brasília (DF); 2012 [cited 2012 Dec 12]. Available from: https://bvsms.saude.gov. br/bvs/saudelegis/cns/2013/res0466_12_12_2012. html

14. Corless IB, Nardi D, Milstead JA, Larson E, Nokes KM, Orsega S, et al. Expanding nursing's role in responding to global pandemics 5/14/2018. Nurs Outlook. 2018;66(4):412-5. DOI: https://doi.org/10.1016/ j.outlook.2018.06.003

15. Cacchione PZ. Moral Distress in the Midst of the COVID-19 Pandemic. Clin Nurs Res. 2020;29(4):215-6. DOI: https://doi.org/10.1177/ 1054773820920385

16. Treston C. COVID-19 in the Year of the Nurse. J Assoc Nurses AIDS Care. 2020;31(3):359-60. DOI: http://dx.doi.org/10.1097/JNC.00000000000 00173 
17. Sun N, Wei L, Shi S, Jiao D, Song R, Ma L, et al. A qualitative study on the psychological experience of caregivers of COVID-19 patients. Am J Infect Control. 2020;48(6):592-8. DOI: https://dx.doi.org/10.1016\%2Fj.ajic.2020.03.018

18. Shen $\mathrm{X}$, Zou $\mathrm{X}$, Zhong $\mathrm{X}$, Yan J, Li L. Psychological stress of ICU nurses in the time of COVID-19. Crit Care. 2020 May;24(200). https:// doi.org/10.1186/s13054-020-02926-2

19. Fuentes PS. Los valores profesionales de las enfermeras (os) chilenas (os) en tiempos de crisis sanitaria por Covid-19. Horiz enferm. 2020;31(1):1-2. DOI: http://dx.doi.org/10.7764/ Horiz_Enferm.31.1.1-3

20. Rodrigues NH, Silva LGA. Gestão da pandemia Coronavírus em um hospital: relato de experiência profissional. J. Nurs Health. 2020;10(spe):e20104004. DOI: https://doi.org/10. 15210/jonah.v10i4.18530

21. Estatela GM, Zabalegui A, Guerra SS. Gestión y liderazgo de los servicios de enfermería en el plan de emergencia de la pandemia COVID-19: la experiencia del Hospital Clínic de Barcelona. Enferm clín. 2020 May 15. DOI: https://doi.org/ doi:10.1016/j.enfcli.2020.05.002

22. Louise DD. What the COVID-19 pandemic tells us about the need to develop resilience in the nursing workforce. Nurs Manag (Harrow). 2020 [cited 2020 May 14];27(3):22-7. Available from: https://pesquisa.bvsalud.org/brasil/resource/pt/ mdl-32400142

23. Adhikari BB, Koonin LM, Mugambi ML, Sliger KD, Washington ML, Kahn EB, et al. Estimating Weekly Call Volume to a National Nurse Telephone Triage Line in an Influenza Pandemic. Health Secur. 2018;16(5):334-40. DOI: https://doi.org/10.1089/hs.2018.0061

24. Williams JL, Mersereau PW, Ruch-Ross H, Zapata LB, Ruhl C. Influenza infection control practices in labor and delivery units during the 2009 H1N1 influenza pandemic. J Obstet
Gynecol Neonatal Nurs. 2013;42(5):527-40. DOI: 10.1111/1552-6909.12243

25. Kennedy MS. Nurses: Courageous, Committed, and Fed Up. Am J Nurs. 2020;120(6):7. DOI: 10.1097/01.NAJ.0000668648.29477.e1

26. Finch J. Legal aspects of COVID-19 pandemic management for community nurses. $\mathrm{Br} \mathrm{J}$ Community Nurs. 2020;25(4):196-9. DOI: https://doi.org/10.12968/bjcn.2020.25.4.196

27. Cipriano PF. 100 years on: the Spanish Flu, pandemics and keeping nurses safe. Int Nurs Rev. 2018;65(3):305-6. DOI: https://doi.org/10.1111/ inr. 12483

28. Campo LA, Puig RC. Enfermeras y practicantes durante la epidemia de gripe de 1918: Análisis a través de la prensa española. Cult cuid. 2019;22(52):109-18. DOI: https://doi.org/10. 14198/cuid.2018.52.10

29. Backes DS, Backes MS, Eedmann AL, Büscher A. O papel profissional do enfermeiro no Sistema Único de Saúde: da saúde comunitária à estratégia de saúde da família. Ciênc saúde coletiva. 2012;17(1):223-30. DOI: http://dx.doi.org/10.1590/ S1413-81232012000100024

30. Coutinho MC. Sentidos do trabalho contemporâneo: as trajetórias identitárias como estratégia de investigação. Cad psicol soc trab [Internet]. 2009 [cited 2020 Jun 20];12(2):189-202. Available from: http://pepsic.bvsalud.org/pdf/ cpst/v12n2/a05v12n2.pdf

31. Araújo-dos Santos T, Silva-Santos H, Silva MN, Coelho ACC, Pires CGS, Melo CMM. Precarização do trabalho de enfermeiras, técnicas e auxiliares de Enfermagem nos hospitais públicos. Rev Esc Enferm USP. 2018;52:e3411 DOI: https://doi. org/10.1590/s1980-220x2017050503411

Recebido: 13 de julho de 2020 Aprovado: 22 de setembro de 2020 Publicado: 22 de novembro de 2021

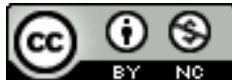

A Revista Baiana de Enfermagem utiliza a Licença Creative Commons - Atribuição-NãoComercial 4.0 Internacional. https://creativecommons.org/licenses/by-nc/4.0/ Este artigo é de acesso aberto distribuído sob os termos da Licença Creative Commons (CC BY-NC). Esta licença permite que outros remixem, adaptem e criem a partir do seu trabalho para fins não comerciais. Embora os novos trabalhos tenham de lhe atribuir o devido crédito e não possam ser usados para fins comerciais, os usuários não têm de licenciar esses trabalhos derivados sob os mesmos termos. 